\title{
Interview
}

\section{Fighting disease from the chromosome end}

Maria A Blasco obtained her PhD in 1993 for her research at the Centro de Biología Molecular 'Severo Ochoa' (Madrid, Spain) under the supervision of M Salas. That same year, Blasco joined the Cold Spring Harbor Laboratory in New York (NY, USA) as a Postdoctoral Fellow under the leadership of CW Greider. In 1997 she returned to Spain to start her own research group at the Centro Nacional de Biotecnología in Madrid. She joined the Centro Nacional de Investigaciones Oncológicas in 2003 as Director of the Molecular Oncology Programme and Leader of the Telomeres and Telomerase Group, and was appointed Centro Nacional de Investigaciones Oncológicas Director in 2011. Blasco has received the Josef Steiner Cancer Research, Rey Jaime I, Körber European Science, Alberto Sols and Fundación Lilly Precilinical Research Awards, as well as the Spanish National Santiago Ramón y Cajal Research Award in Biology (2010). Blasco has also been awarded the EMBO Gold Medal and has served on its Council since 2008.

- Can you please explain a little about your background \& how you became interested in telomere research?

I have always been interested in molecular biology, especially in processes such as aging and cancer, which are responsible for such high financial and human costs in developed countries. During my $\mathrm{PhD}$ thesis at the Universidad Autónoma de Madrid (Madrid, Spain), telomerase, a cellular component closely linked to aging and cancer, started to come to prominence. After finishing my PhD, I moved to the USA to work in Carol W Greider's laboratory at Cold Spring Harbor Laboratory in New York (NY, USA). Greider was a very promising young researcher who had jointly discovered telomerase with her mentor Elizabeth Blackburn, for which they were awarded the Nobel Prize in 2009. Working in Greider's lab was a great opportunity as it allowed me to participate in this emerging and promising field. In the USA, I isolated the mammalian telomerase gene and generated a mouse model that has permitted us to demonstrate its role in aging and cancer.

\section{Why is it important to} understand the regulation of telomeres?

Telomeres protect the chromosome ends, thus preventing chromosomal aberrations that could limit cell viability and cause diseases linked to aging, including cancer. Understanding what molecular mechanisms drive these processes is necessary for the prevention of age-related diseases, such as cancer and cardiovascular diseases, and to identify individuals that may be at risk of disease.

- In your opinion, what is your greatest achievement to date? A researcher is always searching for new discoveries. The search for knowledge never ends, particularly when one is involved in such an exciting and important challenge as fighting cancer. However, if I had to choose, I would go for a 2008 paper published in Cell on the fight against cancer through the activation of biological mechanisms that slow down aging [1]. This was the first demonstration in any organism of the role of telomerase in delaying aging.

\section{- Can you explain a little about} what you are currently working on? Currently, one of my main interests is to understand the potential use of telomerase activation in preventing diseases related to aging, including cancer. I am very proud of showing that telomerase activation by means of gene therapy is sufficient to delay aging and increase longevity in the absence of further cancer [2]. These findings identify telomerase and telomeres as one of the molecular mechanisms underlying aging [3].

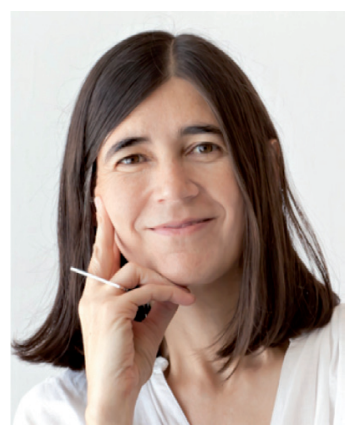

\section{Maria A Blasco}

Spanish National Cancer Research Centre (CNIO), 3 Melchor Fernandez Almagro, 28029, Madrid, Spain mblasco@cnio.es 


\section{"Understanding the role of epigenetics in major human diseases to me still remains as one of the main interests of this field"}

- One of your many research achievements was the finding that mammalian telomeres \& subtelomeres have epigenetic marks characteristic of constitutive heterochromatin. Can you tell us a little about these findings \& the research that went into producing them?

We found that telomeres and subtelomeric chromatin are similar to pericentromeric repairs regarding the fact that both are enriched in heterochromatic marks. We also found that altering chromatin and DNA modifications at telomeres and subtelomeres represents a higher-order control of telomere length. We have dissected this by using mutant cells for the different histone methyltranferases and DNA methylases. Now we now that this process may be relevant for cancer, since cancer cells have alterations in subtelomeric methylation, which, in turn, would favor telomere elongation by the action of telomerase and by increasing homologous recombination between telomeric sequences.

More recently, we also found that these epigenetic modifications are important for telomere elongation, associated with induction of pluripotency in induced pluripotent stem cells. In particular, induction of pluripotency by defined factors results in loss of heterochromatic marks at telomeres, which is concomitant with increased telomeric transcription (the so-called long noncoding telomeric RNAs), as well as dramatic net telomere elongation and increased telomeric recombination.

- What did this research tell us about the importance of histone modifications on the counting of telomere repeats?

In addition to the results described above, we also observed that when telomeres become short, there is a decrease in the density of heterochromatic marks at telomeres and subtelomeres, which, in turn, could facilitate a higher access for telomerase and other telomere elongation mechanisms (i.e., homologous recombination) to the telomeres. This could be a possible mechanism to explain the known fact that telomerase preferentially elongate short telomeres in mammals [4].
One of your publications suggests a role for mammalian DNA methyltransferases in telomere length control. Can you please explain a little about this research $\&$ the outcomes?

Briefly, loss of DNA methylation in subtelomeric repeats results in increased telomere length, suggesting that this mark acts as a negative regulator of telomere length.

Another publication suggests that miRNA may have a role in the control of DNA methylation \& telomere recombination. Can you explain a little about this relationship?

This was found by studying cells deficient for DICER, an enzyme that is essential for the generation of miRNAs. We observed that the telomeres of these cells were abnormally long, and we could demonstrate that this was the result of deregulation of a cluster of miRNAs which regulated the Rb family of proteins, which we had also previously shown to regulate telomere length.

These studies were mainly conducted in mice. Has this translated into any studies of human cells since?

As mentioned above, we demonstrated that human cancer cell lines that have hypomethylated subtelomeric repeats are also the ones that have longer telomeres, as well as an increased frequency of recombination between telomeric sequences (another way to elongate telomeres, known as 'alternative lengthening of telomeres').

How have these findings aided the understanding of aging?

Epigenetic alterations and telomere length are two of the hallmarks of aging [3]. It is very likely that, based on the studies carried out in mice and cell lines, epigenetic abnormalities can also influence aging through their impact on the regulation of telomeric and subtelomeric chromatin.

Could the understanding of the epigenetic regulation of telomeres translate into any therapeutic avenues, such as for aging disorders or cancer, in the future?

The results obtained in mice and human cells suggest that drugs targeting DNA 
methyltransferases or histone methyltransferases have an impact on telomere length regulation, by making telomeres longer. The only problem I see is that inhibiting these enzymes has many other effects because they target many more genomic regions than just telomeres and subtelomeres.

What, in your opinion, is the most exciting research currently being conducted in epigenetics? I think that the ENCODE project is pretty exciting. Having an interest in aging, I find that it is also extremely interesting to determine the epigenetic control of aging.

What do you think will be the main advances in this field in the next 10 years?

Understanding the role of epigenetics in major human diseases to me still remains as one of the main interests of this field.

\section{Disclaimer}

The opinions expressed in this interview are those of the interviewee and do not necessarily reflect the views of Future Medicine Ltd.

\section{Financial \& competing interests disclosure}

MA Blasco has no relevant affiliations or financial involvement with any organization or entity with a financial interest in or financial conflict with the subject matter or materials discussed in the manuscript. This includes employment, consultancies, honoraria, stock ownership or options, expert testimony, grants or patents received or pending, or royalties.

No writing assistance was utilized in the production of this manuscript.

\section{References}

1 Tomás-Loba A, Flores I, Fernández-Marcos PJ et al. Telomerase reverse transcriptase delays aging in cancer-resistant mice. Cell 135(4), 609-622 (2008).

2 Bernardes de Jesus B, Vera E, Schneeberger K et al. Telomerase gene therapy in adult and old mice delays aging and increases longevity without increasing cancer. EMBO Mol. Med. 4(8), 691-704 (2012).

3 López-Otín C, Blasco MA, Partridge L, Serrano M, Kroemer G. The hallmarks of aging. Cell 153(6), 1194-1217 (2013).

4 Samper E, Flores JM, Blasco MA. Restoration of telomerase activity rescues chromosomal instability and premature aging in $\operatorname{Terc}^{-1-}$ mice with short telomeres. EMBO Rep. 2(9), 800-807 (2001). 